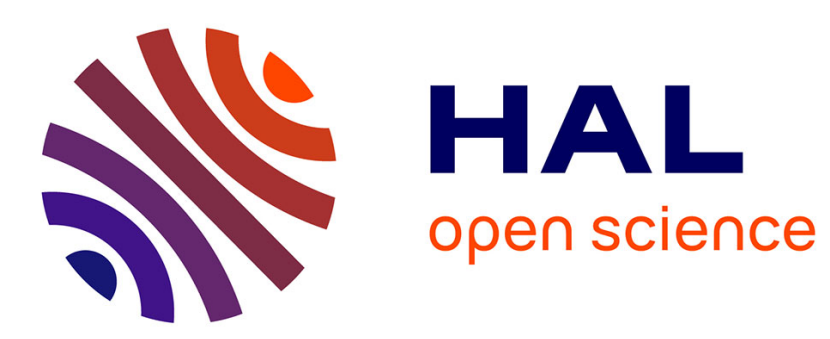

\title{
In-plane backward and zero group velocity guided modes in rigid and soft strips
}

Jérôme Laurent, Daniel Royer, Claire Prada

\section{To cite this version:}

Jérôme Laurent, Daniel Royer, Claire Prada. In-plane backward and zero group velocity guided modes in rigid and soft strips. Journal of the Acoustical Society of America, 2020, 147 (2), pp.1302-1310. $10.1121 / 10.0000760$. hal-03082256

\section{HAL Id: hal-03082256 https://hal.science/hal-03082256}

Submitted on 5 Oct 2021

HAL is a multi-disciplinary open access archive for the deposit and dissemination of scientific research documents, whether they are published or not. The documents may come from teaching and research institutions in France or abroad, or from public or private research centers.
L'archive ouverte pluridisciplinaire HAL, est destinée au dépôt et à la diffusion de documents scientifiques de niveau recherche, publiés ou non, émanant des établissements d'enseignement et de recherche français ou étrangers, des laboratoires publics ou privés. 
In-plane backward and zero group velocity guided modes in rigid and soft strips Jérôme Laurent, Daniel Royer, and Claire Prada

Citation: The Journal of the Acoustical Society of America 147, 1302 (2020); doi: 10.1121/10.0000760 View online: https://doi.org/10.1121/10.0000760

View Table of Contents: https://asa.scitation.org/toc/jas/147/2

Published by the Acoustical Society of America

JASA 


\title{
In-plane backward and zero group velocity guided modes in rigid and soft strips
}

Jérôme Laurent, Daniel Royer, and Claire Prada ${ }^{a}$

Ecole Supérieure de Physique et de Chimie de la ville de Paris, Paris Sciences et Lettres University, Centre National de la Recherche Scientifique, Institut Langevin, 1 rue Jussieu, Paris, France

\begin{abstract}
:
Elastic waves guided along bars of rectangular cross sections exhibit complex dispersion. This paper studies in-plane modes propagating at low frequencies in thin isotropic rectangular waveguides through experiments and numerical simulations. These modes result from the coupling at the edge between the first order shear horizontal mode $S H_{0}$ of phase velocity equal to the shear velocity $V_{T}$ and the first order symmetrical Lamb mode $S_{0}$ of phase velocity equal to the plate velocity $V_{P}$. In the low frequency domain, the dispersion curves of these modes are close to those of Lamb modes propagating in plates of bulk wave velocities $V_{P}$ and $V_{T}$. The dispersion curves of backward modes and the associated zero group velocity $(\mathrm{ZGV})$ resonances are measured in a metal tape using noncontact laser ultrasonic techniques. Numerical calculations of in-plane modes in a soft ribbon of Poisson's ratio $\nu \approx 0.5$ confirm that, due to very low shear velocity, backward waves and ZGV modes exist at frequencies that are hundreds of times lower than ZGV resonances in metal tapes of the same geometry. The results are compared to theoretical dispersion curves calculated using the method provided in Krushynska and Meleshko [J. Acoust. Soc. Am. 129, 1324-1335 (2011)].
\end{abstract} (C) 2020 Acoustical Society of America. https://doi.org/10.1121/10.0000760

(Received 5 November 2019; revised 29 January 2020; accepted 1 February 2020; published online 26 February 2020)

[Editor: Michael R. Haberman]

Pages: $1302-1310$

\section{INTRODUCTION}

Early studies on the propagation of elastic waves in thin elongated strips were motivated by the need for long time ultrasonic delay lines. Today, rectangular elastic waveguides are encountered in miscellaneous contexts at different scales, from civil engineering structures to cantilever, micro-electromechanical systems (MEMS), or even in nanotechnology. ${ }^{1,2}$ Thin elastic wave guides are also present in biological systems as, for example, tendons and ligaments in the musculoskeletal system ${ }^{3}$ or the basilar and tectorial membranes in the cochlea. ${ }^{4}$ Elastic waves propagation in these elongated structures is quite complex, and several modes can propagate with interesting dispersion properties that strongly depend on the material Poisson's ratio. In particular, some modes exhibit negative dispersion.

The existence of backward elastic modes propagating in homogeneous waveguides is well known and has been put in evidence in the past century. ${ }^{5,6}$ Experimental observations of these modes were first reported in elastic cylinders and plates. ${ }^{7-9}$ Today, these backward modes can be clearly observed using laser ultrasonics techniques ${ }^{10}$ and also in piezoelectric plates, using interdigital transducers. ${ }^{11}$ At the minimum frequency of a backward mode, the resonance associated to the zero group velocity (ZGV) mode was pointed out by Tolstoy and Usdin ${ }^{12}$ and observed by Holland and Chimenti using air coupled transducers. ${ }^{13} \mathrm{ZGV}$ resonances can be measured in piezoelectric plates up to $\mathrm{GHz}$ frequencies. ${ }^{14}$ Noncontact laser ultrasonic techniques allow precise measurements and analyses of these

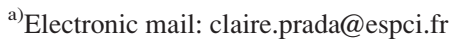

local resonances in plates ${ }^{10,15-18}$ but also in circular hollow cylinders ${ }^{19,20}$ and rods. ${ }^{21,22}$

While elastic guided modes in isotropic plates and circular cylinders are well described by an analytical formulation of the dispersion equations, the theory of elastic rectangular waveguides is much more complex. Indeed, the modes cannot be expressed in a closed form, except for some particular width-to-thickness ratios that were determined by Mindlin and Fox. ${ }^{23}$ Since the seminal work of Morse, ${ }^{24}$ several low frequency approximations were proposed, for example, Medick used a one-dimensional quadratic approximation allowing the calculation of the first eight breathing modes. ${ }^{25}$ More recently, several studies based on numerical simulations using finite and boundary element methods provided approximate dispersion curves. ${ }^{26-31} \mathrm{~A}$ complete review of the theory can be found in the papers by Meleshko and Krushynska, ${ }^{32,33}$ where the dispersion equations of rectangular elastic bars are formulated as infinite series, and normal modes are studied for various width-to-thickness ratios and material parameters. The existence of several backward modes for Poisson's ratio up to 0.5 was pointed out for bars of width-to-thickness ratios higher than five in the study by Krushynska et al. (Sec. III of Ref. 33).

In addition to these theoretical studies, the large majority of experimental results found in the literature deals with flexural modes at low frequencies. Seventy years ago, Morse measured dispersion curves on bars of different width-to-thickness ratios and compared them with low frequency approximation. ${ }^{34}$ Twenty years later, the dispersion curves of higher order modes, including backward modes, were measured by Hertelendy in a square bar of an 
aluminum alloy. ${ }^{35}$ More recently, several dispersion curves were measured in wooden bars by Veres et al. using laser interferometry and then compared to numerical simulations of a wooden orthotropic beam. ${ }^{36}$ More recently, Serey et al. addressed the selective generation of pure guided waves in a thin metal bar of rectangular cross section using three component interferometry and an array of transducers in a frequency range below the shear cutoff frequency. ${ }^{37}$ They were able to detect and generate modes with dominant out of plane displacement corresponding to edge reflections of the $A_{0}$ Lamb mode. In picosecond acoustics, Jean et al. have measured elastic resonances and a backward mode in a gold nano-beam of rectangular cross section (370-nm wide and $110-n m$ thick). With semi-analytical finite element simulations (SAFE), the backward wave was identified as the first dilatational mode. ${ }^{2}$ Very few studies report on measurements of in-planes modes. Cegla et al. detected a shear horizontal mode propagating in a steel tape with a dual laser probe at a frequency far above the shear cutoff frequency, where the mode is nondispersive and propagates at the shear wave velocity. ${ }^{30}$ In-plane guided waves were also observed by Sellon et al. in ex vivo tectorial membranes using optical means. 4

In the present paper, we consider the low frequency inplane modes propagating in thin rectangular bars. We study the corresponding backward mode and associated ZGV resonances for metal tapes as well as soft ribbons. The low frequency in-plane guided modes of rectangular tapes are described in Sec. II. The dispersion curves are calculated for a metal and a soft strip following Krushynska et al. ${ }^{33}$ and compared to the corresponding Rayleigh-Lamb (RL) modes approximation. In Sec. III, ZGV resonances and dispersion curves of in-plane modes measured using laser ultrasonic techniques are presented and compared to the theory. In Sec. IV, comparison between modes propagating in hard and soft ribbons is achieved through numerical simulations.

\section{IN-PLANE MODES OF A THIN RECTANGULAR BEAM}

At low frequencies, an isotropic plate supports the propagation of only three modes: the shear horizontal mode $\mathrm{SH}_{0}$, the compressional symmetric $S_{0}$, and the flexural antisymmetric $A_{0}$ Lamb modes. The symmetrical $S H_{0}$ and $S_{0}$ modes are both in-plane and nondispersive: While the phase velocity of $S_{0}$ is the shear velocity $V_{T}$, the $S_{0}$ phase velocity is approximated by the plate velocity $V_{P}$, expressed from the bulk velocities $V_{L}$ and $V_{T}$, as

$$
V_{P}=2 V_{T} \sqrt{1-\frac{V_{T}^{2}}{V_{L}^{2}}} .
$$

This approximation is valid for frequencies $f$ smaller than the first shear cutoff frequency $V_{T} /(2 d)$, where $d$ is the plate thickness. $^{38}$

If the plate is bounded in the transverse dimension, thus forming a rectangular waveguide of width $b \gg d$, then the in-plane modes $S H_{0}$ and $S_{0}$ are coupled through reflections at the edges (Fig. 1). As shown by Morse ${ }^{24}$ and Medick and $\mathrm{Pao},{ }^{39}$ this coupling is very similar to the coupling of shear and compressional bulk waves in a plate. The resulting guided modes have dispersion curves close to those of Lamb modes in a plate of thickness $b$ and bulk wave velocities $V_{P}$ and $V_{T}$, which correspond to a material of Poisson's ratio

$$
\nu^{\prime}=\frac{\nu}{1+\nu}
$$

where $\nu$ is the Poisson's ratio of the beam material.

The calculation of guided modes in a rectangular waveguide is described in Krushynska and Meleshko. ${ }^{33}$ As in the earlier paper by Fraser, ${ }^{40}$ the modes are classified in four families depending on the displacement symmetries: the longitudinal $L$-modes, the torsional $T$-modes, the bending $B_{x}$-modes, and $B_{y}$-modes. For thin beams with the smallest dimension along the $y$ axis, the symmetrical in-plane $S^{\prime}$-modes correspond to the longitudinal $L$-modes, and the antisymmetrical in-plane $A^{\prime}$-modes correspond to the bending $B_{x}$-modes. In Ref. 33, the low frequency approximation of in-plane modes was confirmed for a plate of width-to-thickness ratio $b / d=5$ and Poisson's ratio $\nu=1 / 4$, and for frequencies up to four times the first cutoff frequency $V_{T} /(2 b)$.

The first symmetrical mode, here denoted $S_{0}^{\prime}$, is nondispersive below the first cutoff frequency and has a phase velocity $V_{P}^{\prime}$ slightly lower than the plate velocity $V_{P}$ and equal to

$$
V_{P}^{\prime}=2 V_{T} \sqrt{1-\frac{V_{T}^{2}}{V_{P}^{2}}}
$$

Using the plate to shear velocity ratio

$$
\frac{V_{P}}{V_{T}}=\sqrt{\frac{2}{1-\nu}}=\sqrt{\frac{2\left(1-\nu^{\prime}\right)}{1-2 \nu^{\prime}}},
$$

a remarkably simple formulation of the pseudo-plate velocity to shear velocity ratio is obtained

$$
\frac{V_{P}^{\prime}}{V_{T}}=\sqrt{\frac{2}{1-\nu^{\prime}}}=\sqrt{2(1+\nu)} .
$$

In fact, the velocity $V_{P}^{\prime}$ is the bar velocity $\sqrt{E / \rho}$, which is the velocity of the low frequency longitudinal mode in an elastic guide of finite cross section as established by Lord Rayleigh. ${ }^{41}$ Noteworthy simple expressions of the velocities

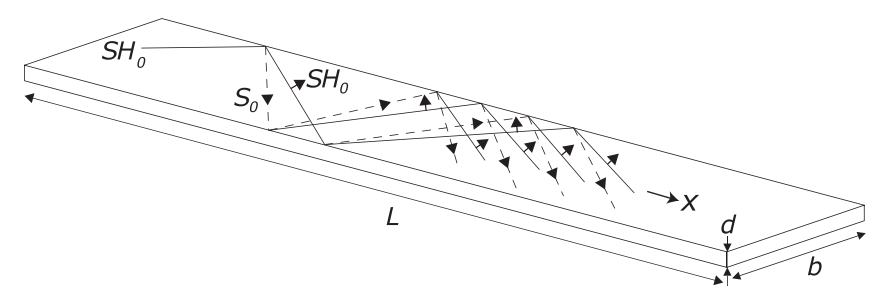

FIG. 1. In-plane modes conversion in a thin rectangular beam. 
TABLE I. Remarkable plate and bar velocities.

\begin{tabular}{lcccc}
\hline \hline Material & $\nu$ & $\nu^{\prime}$ & $V_{P}$ & $V_{P}^{\prime}$ \\
\hline Metal & $\frac{1}{3}$ & $\frac{1}{4}$ & $\sqrt{3} V_{T}$ & $\sqrt{\frac{8}{3}} V_{T}$ \\
Soft & 1 & 1 & & $\sqrt{3} V_{T}$ \\
\hline \hline
\end{tabular}

are found for two particular cases. For $\nu=1 / 3$, the plate velocity is $V_{P}=\sqrt{3} V_{T}$, and then for $\nu^{\prime}=1 / 4$, the velocity of the $S_{0}^{\prime}$ mode at low frequencies is $V_{P}^{\prime}=\sqrt{8 / 9} V_{P}$ $\approx 0.94 V_{P}$. For soft materials, $\nu$ approaches 0.5 , and the plate velocity is $V_{P}=2 V_{T}$, which is much smaller than the longitudinal velocity. ${ }^{42}$ Thus, in soft ribbon the bar velocity is $V_{P}^{\prime}=\sqrt{3} V_{T}$. These remarkable parameters are gathered in Table I. Here, using the Meleshko-Krushynska (MK) model proposed in Ref. 33, theoretical dispersion curves are calculated for a duraluminum tape of thickness $d=2 \mathrm{~mm}$, width $b=30 \mathrm{~mm}$, and bulk velocities $V_{L}=6400 \mathrm{~m} / \mathrm{s}, V_{T}$ $=3140 \mathrm{~m} / \mathrm{s}$. In Fig. 2(a), the normalized frequency $\mathrm{fb} / V_{T}$ is represented as a function of the dimensionless wave number $k b$. Lamb modes in a plate of bulk velocities $V_{T}$ and $V_{P}$ and thickness $b$ are also displayed for comparison. A very good agreement with the Lamb modes approximation is observed for frequencies up to $f=4 V_{T} / b$. As in a plate, several backward and ZGV modes exist. They occur when a shear (or $S H_{0}$ ) cutoff frequency $n V_{T} / b$ is close to a plate mode (or $S_{0}$ ) cutoff frequency $m V_{P} / b$ of the same symmetry. $S_{1}^{\prime} S_{2}^{\prime}$ - and
$A_{2}^{\prime} A_{3}^{\prime}-\mathrm{ZGV}$ points are found at frequency wave number $\left(f b / V_{T}, k b\right)=(0.824,1.77)$ and $(1.49,1.95)$, respectively [Fig. 2(a)]. The thick solid lines represent the first modes $A_{0}$, $S_{0}$, and $S H_{0}$ of an unbounded plate of thickness $d$. For higher frequencies between $A_{0}$ and $S H_{0}$ modes the $A^{\prime}{ }_{0}$ and $S_{0}^{\prime}$ modes converge toward the edge or pseudo-Rayleigh mode.

While in plates, the $S_{1} S_{2}-Z G V$ Lamb mode only exits for Poisson's ratio below $0.451,{ }^{43}$ in thin rectangular beams, the $S_{1}^{\prime} S_{2}^{\prime}-\mathrm{ZGV}$ mode exists for any Poisson's ratio. The behaviour of in-plane modes is particularly interesting in the case of soft material. Indeed, for $\nu \approx 0.5$, the plate velocity $V_{P}$ is equal to $2 V_{T}$, which results in a Dirac cone at the frequency $f=V_{T} / b$, as it happens for Lamb modes in a plate of Poisson's ratio $\nu=1 / 3 .^{5,6,44,45}$ This is illustrated in Fig. 2(b), which displays the in-plane mode dispersion curves for a 2-mm-thick and 20-mm-wide tape of velocities $V_{L}=1500 \mathrm{~m} / \mathrm{s}$ and $V_{T}=5 \mathrm{~m} / \mathrm{s}(\nu=0.4998)$. Such low shear velocity corresponds to typical velocities measured in agar gel or soft tissues. The second $S H_{0}$ cutoff frequency coincides with the first $S_{0}$ cutoff frequency $f=V_{P} /(2 b)$, and the repulsion between the branches $S_{1}^{\prime}$ and $S_{2}^{\prime}$ results in a $8 \%$ relative bandwidth $S_{2 b}^{\prime}$ backward branch (from 230 to $249 \mathrm{~Hz}$ in the presented case). For both branches, the group velocity remains finite as $k \rightarrow 0$ and converges to $V_{g}$ $=2 V_{T} / \pi .^{5,6}$ The $S_{1}^{\prime} S_{2}^{\prime}-\mathrm{ZGV}$ mode occurs at a frequency $\times$ width product $f b=4.53 \mathrm{kHz} \mathrm{mm}$, which is more than 2 orders of magnitude lower than the frequency $\times$ width product of the same $\mathrm{ZGV}$ mode in a metal tape, $f b$ $=2.64 \mathrm{MHz} \mathrm{mm}$.
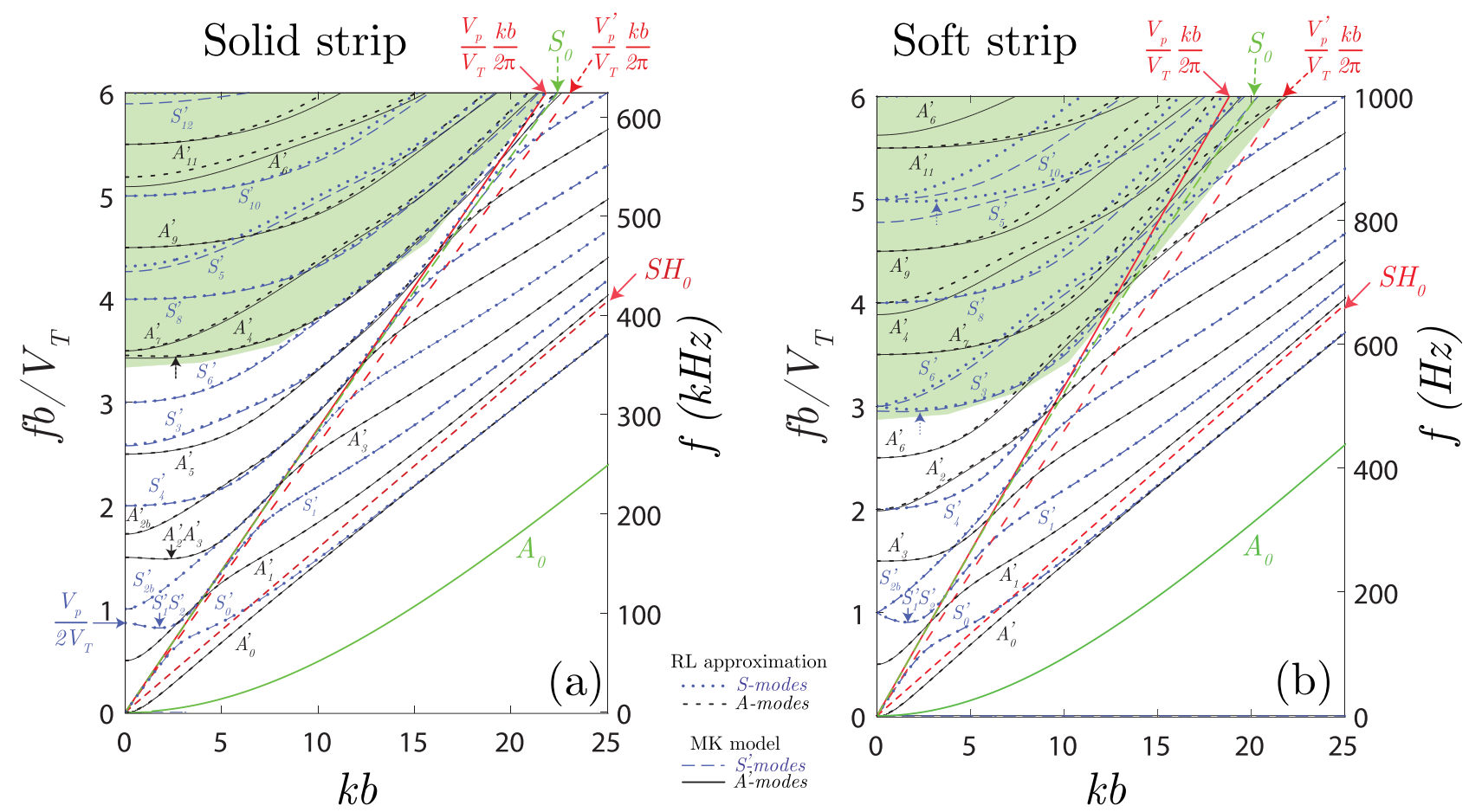

FIG. 2. (Color online) In-plane mode dispersion curves calculated with the MK model for solid and soft strips of width $b=30 \mathrm{~mm}$, thickness $d=2 \mathrm{~mm}$, and Poisson's ratio $\nu$, compared to the RL modes for a plate of thickness $b$ and Poisson's ratio $\nu^{\prime}=\nu /(1+\nu)$. Thick red, blue, and green solid lines are the first two Lamb modes and the first shear mode of a plate of thickness $d$ and Poisson's ratio $\nu$, respectively, and the red dashed line indicates the plate velocity. (a) Duraluminum tape of Poisson's ratio $\nu=0.34$, (b) soft ribbon of bulk wave velocities $V_{L}=1500 \mathrm{~m} / \mathrm{s}$ and $V_{T}=5 \mathrm{~m} / \mathrm{s}$. The cutoff frequency of the $S_{1}^{\prime}$ mode is $f b=V_{T}$. The $S_{0}$ mode coincides with the straight line $2 \pi f=V_{P} k$, while the $S_{0}^{\prime}$ mode is close to the line $2 \pi f=V_{P}^{\prime} k$ for $f b<0.4 V_{T}$. 


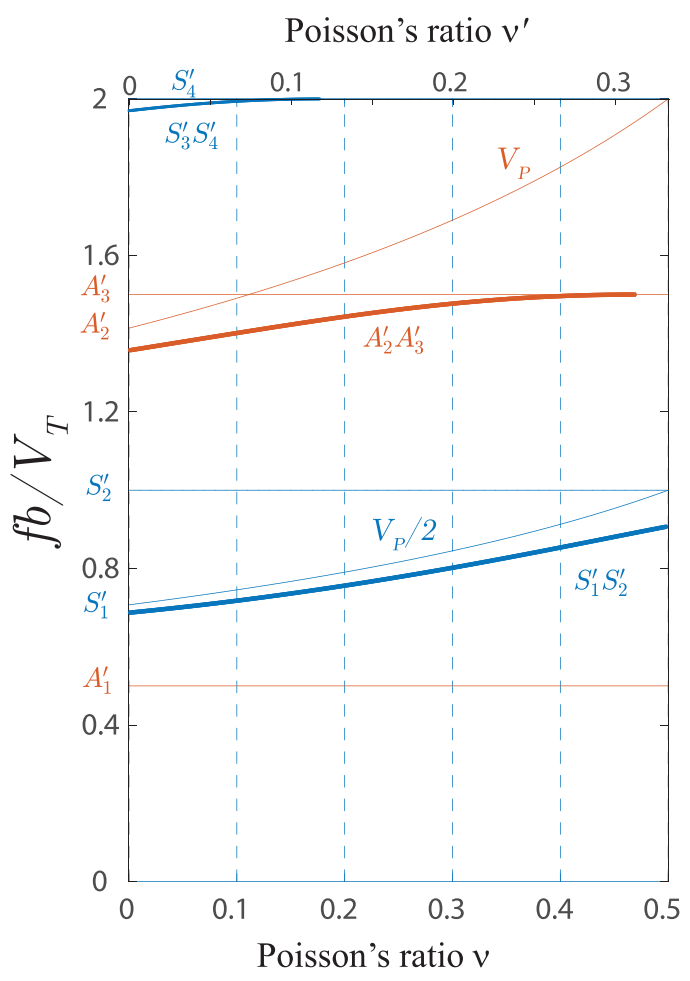

(a)

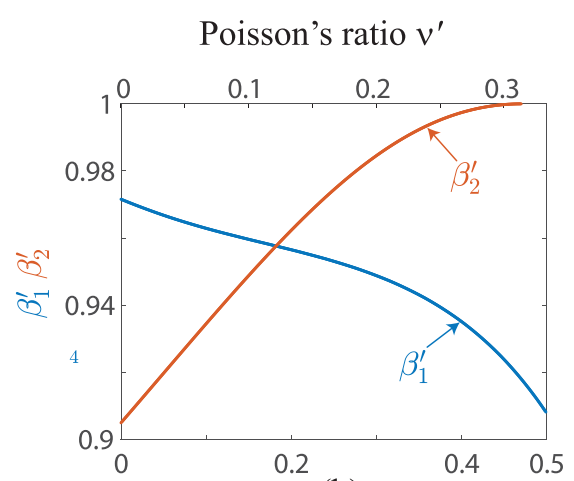

(b)

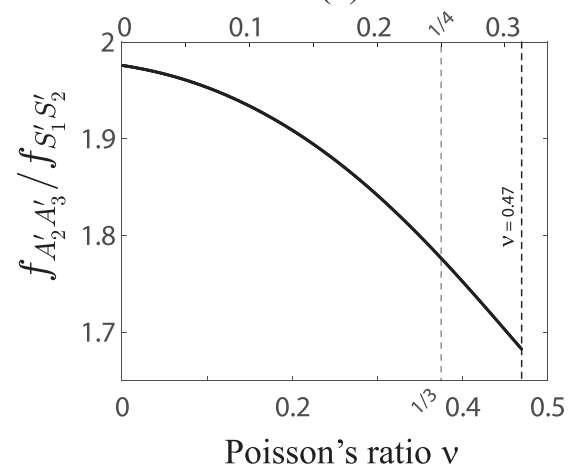

(c)

FIG. 3. (Color online) In-plane modes of a thin strip. (a) Normalized cutoff (thin lines) and ZGV frequencies (thick lines) as a function of the material Poisson's ratio; the thin horizontal lines correspond to $S H_{0}$ cutoff frequencies. (b) Variations of the resonance parameters $\beta_{1}^{\prime}$ and $\beta_{2}^{\prime}$ versus Poisson's ratio. (c) Ratio between $A_{2}^{\prime} A_{3}^{\prime}$ - and $S_{1}^{\prime} S_{2}^{\prime}$-ZGV frequencies.

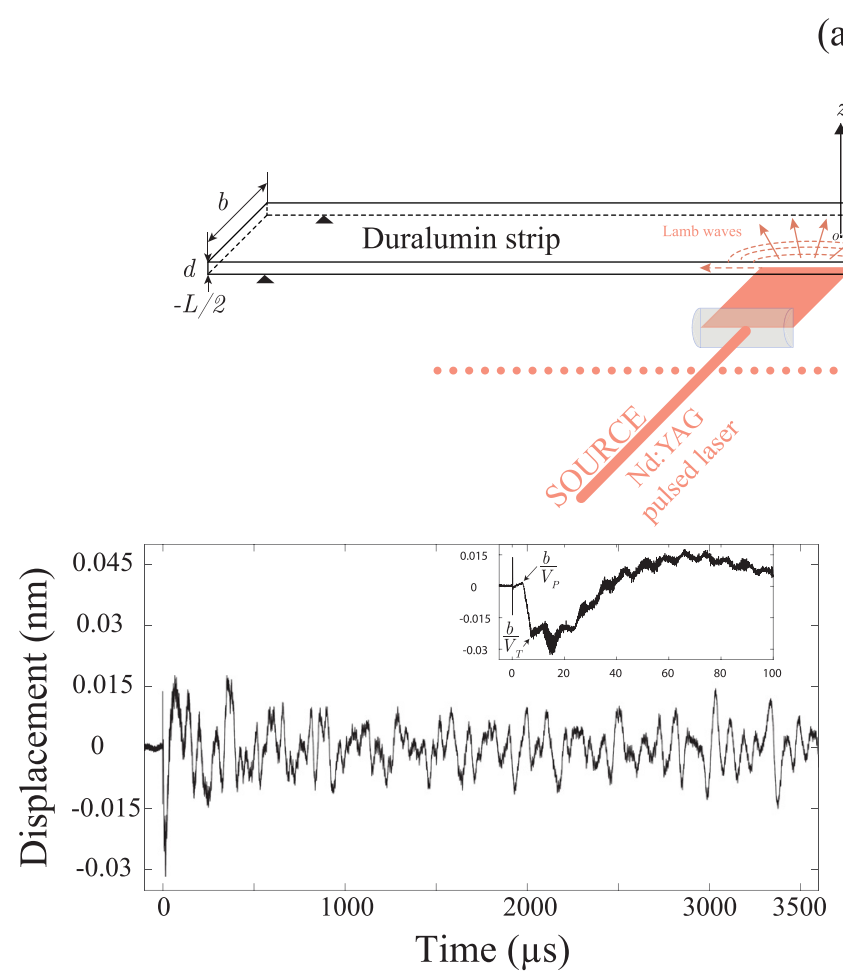

(b) (a)
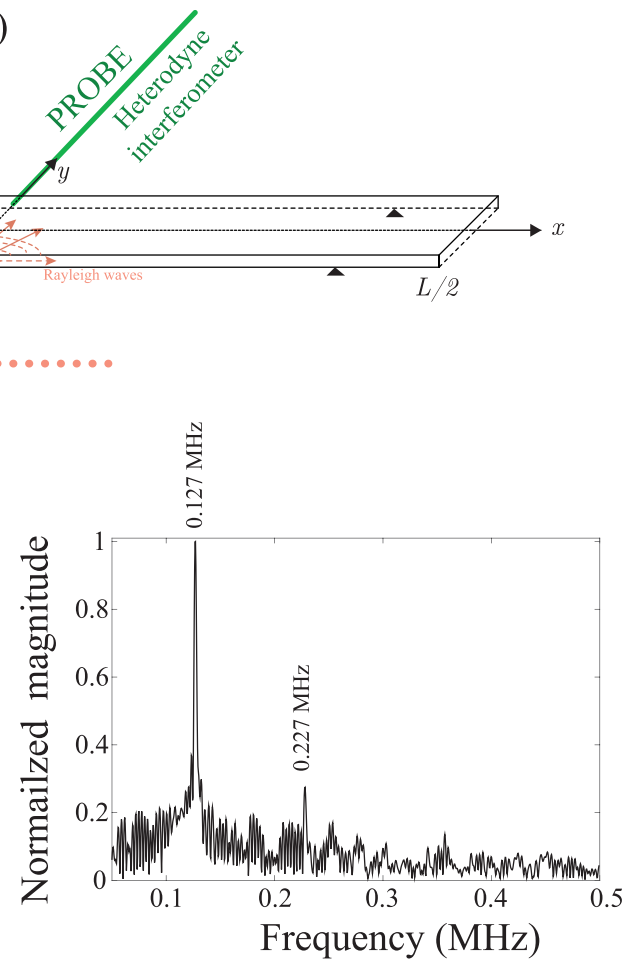

(c)

FIG. 4. (Color online) (a) Schematic diagram of an in-plane guided wave measurement in a metallic strip. The excitation is achieved on one edge with a laser line source parallel to the plate surface. The normal surface displacement is measured on the opposite edge with an interferometer. (b) Displacement is measured at the epicenter on a strip of thickness $d=0.5 \mathrm{~mm}$ and width $b=20 \mathrm{~mm}$. (c) Fourier transform of the first $200 \mu \mathrm{s}$ of the measured signal. 
As for plates or cylinders, the existence of $\mathrm{ZGV}$ modes depends on the material elastic parameters. For isotropic thin rectangular beams, this dependence is illustrated in Fig. 3(a), which displays cutoff and ZGV resonance frequencies versus Poisson's ratio. Horizontal and increasing curved thin lines correspond to $\mathrm{SH}_{0}$ and $\mathrm{S}_{0}$ width resonances, respectively. It appears that while the $S_{1}^{\prime} S_{2}^{\prime}$-ZGV mode exists for all Poisson's ratio, the $A_{1}^{\prime} A_{2}^{\prime}$ ZGV mode exists for Poisson's ratio $\nu$ up to 0.47 . Using the approach proposed in Clorennec et al. for plates, ${ }^{19}$ the resonance parameter $\beta_{1}^{\prime}$ is defined as the ratio of the $S_{1}^{\prime} S_{2}^{\prime}-\mathrm{ZGV}$ frequency to the first $S_{0}$ cutoff frequency $V_{P} /(2 b)$, and $\beta_{2}^{\prime}$ is defined as the ratio of the $A_{1}^{\prime} A_{2}^{\prime}-\mathrm{ZGV}$ frequency to the third $S H_{0}$ cutoff frequency $3 V_{T} /(2 b)$. There is a one-to-one correspondence between the ratio of the first two $\mathrm{ZGV}$ resonances $3 \beta_{2}^{\prime} V_{T} /\left(\beta_{1}^{\prime} V_{P}\right)$ and the Poisson's ratio [Fig. 3(a)]. As a consequence, and similar to the plates, the measurement of the first two local resonances provides the material Poisson's ratio of the rectangular tape.

\section{MEASUREMENTS ON A METAL TAPE}

In-plane guided modes are observed on metal strips using noncontact laser ultrasonic techniques. A Q-switched $\mathrm{Nd}$ :YAG (yttrium aluminum garnet) laser providing 8-ns pulses of $10-\mathrm{mJ}$ energy at a $8-\mathrm{Hz}$ repetition rate (Quantel Centurion, Quantel SA, Les Ulis, France) is used as a thermoelastic source. The laser beam of diameter $2.5 \mathrm{~mm}$ is focused into a narrow line with a beam expander $(\times 4)$ and a cylindrical lens (focal length $250 \mathrm{~mm}$ ). The full length of the source at $1 / e$ of the maximum value was found to be $10 \mathrm{~mm}$, and the width was estimated to be $0.3 \mathrm{~mm}$. With these features, the absorbed power density was below the ablation threshold. The source length is smaller than the optimal length to generate a $\mathrm{ZGV}$ mode but reasonable to generate several in-plane modes. ${ }^{46}$

In order to generate modes with symmetrical displacement component $u_{z}$, the line source was located in the middle of the tape edge (plane $z=0$ ). Then, to detect in-plane modes, the normal surface displacement $u_{y}$ was measured on the opposite edge with a heterodyne interferometer

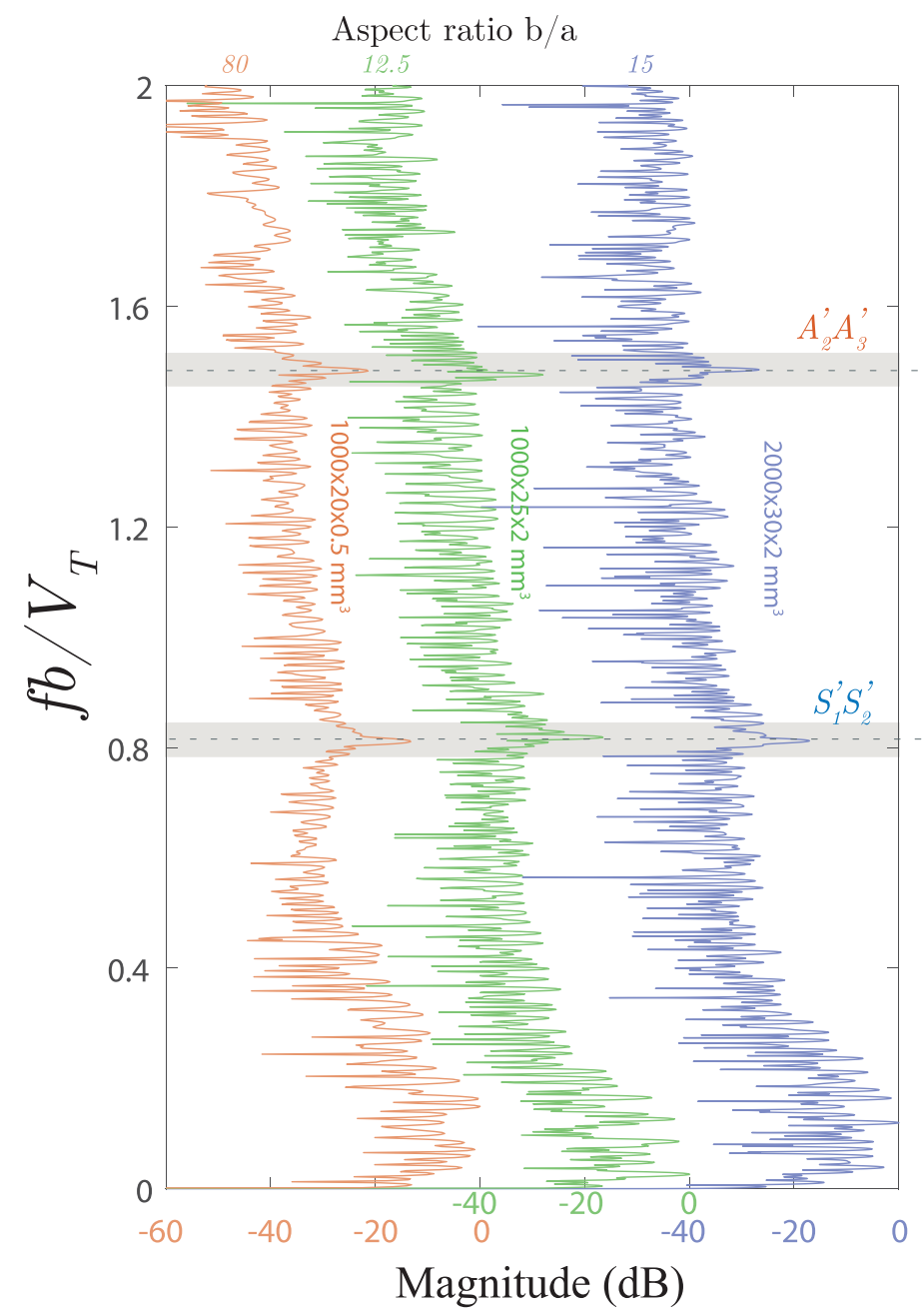

(a)

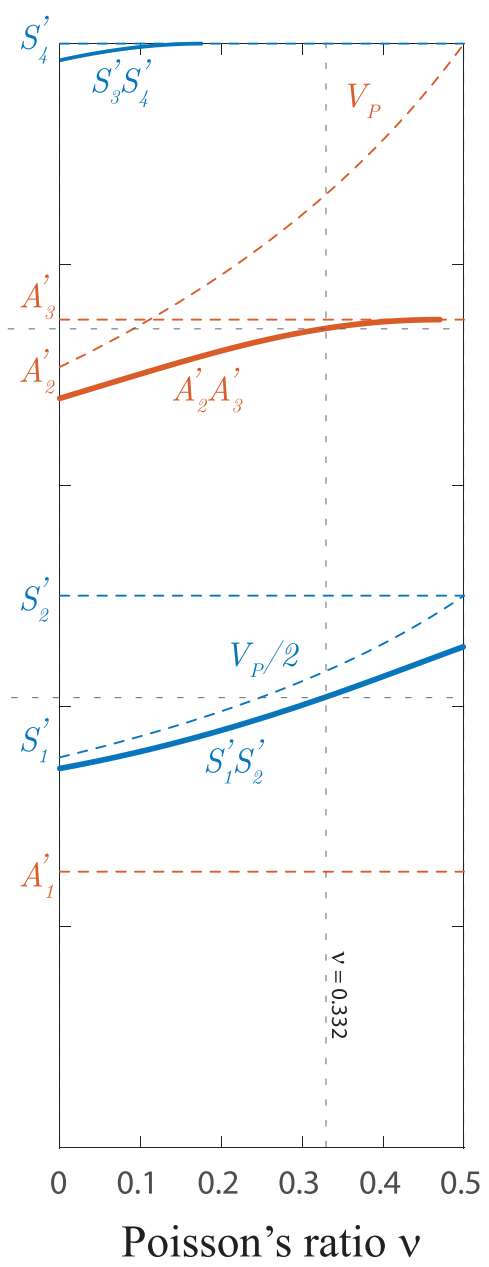

(b)

FIG. 5. (Color online) (a) Local resonance spectrum measured on three duraluminum tapes. (b) ZGV (solid lines) and cutoff (dashed lines) normalized resonance frequencies as a function of Poisson's ratio. 


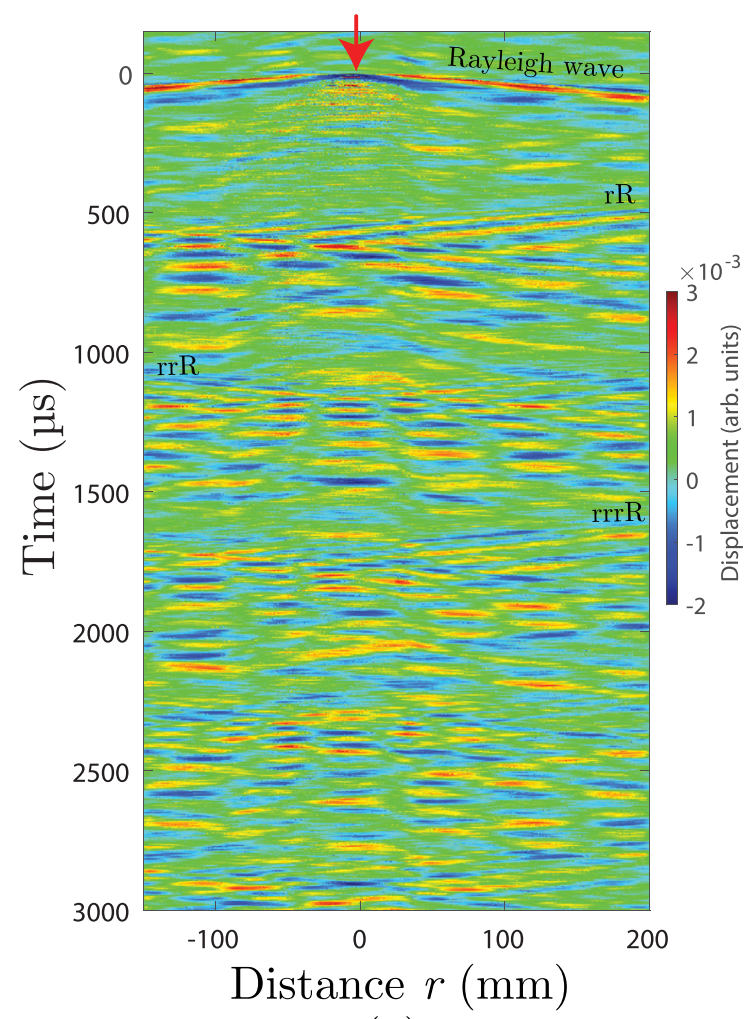

(a)

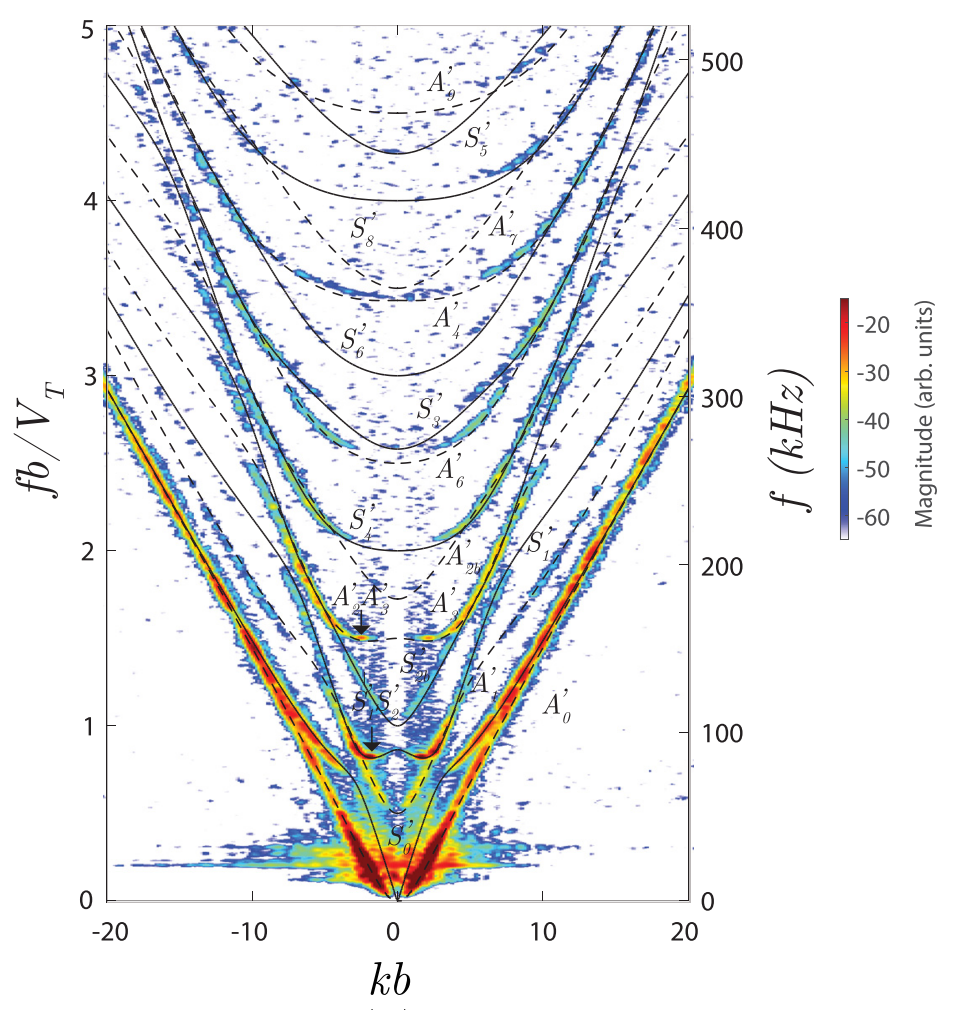

(b)

FIG. 6. (Color online) (a) Displacement $u_{y}$ measured along the edge of a duraluminum tape of dimensions $L=2 \mathrm{~m}, b=30 \mathrm{~mm}$, and $d=2 \mathrm{~mm}$. (b) Twodimensional (2D)-Fourier analysis of the displacement field with superimposed theoretical dispersion curves in black and red dashed lines.

equipped with a 100-mW frequency doubled Nd:YAG laser [optical wavelength $\Lambda=532 \mathrm{~nm}$; Fig. 4(a)].

First measurements were done on a 1 -m-long duraluminum tape of thickness $d=0.5 \mathrm{~mm}$ and width $b=20 \mathrm{~mm}$. A significant displacement is detected for more than $3 \mathrm{~ms}$ after the laser impact [Fig. 4(b)]. This duration is mostly due to the reflections at the tape ends of edge modes similar to those measured by Jia et al. ${ }^{47}$ During the first $100 \mu$ s (inset) a typical waveform is observed with the first arrival of the compressional mode $S_{0}$, followed by the first arrival of the shear mode $\mathrm{SH}_{0}$, as well as a low frequency resonance. The Fourier transform displayed in Fig. 4(c) highlights the first two ZGV resonances: $S_{1}^{\prime} S_{2}^{\prime}$ at $0.127 \mathrm{MHz}$ and $A_{2}^{\prime} A_{3}^{\prime}$ at $0.227 \mathrm{MHz}$.

Similar measurements were also performed on two $d=2$-mm-thick tapes of width $b=25$ or $30 \mathrm{~mm}$. The normalized spectrum is displayed in Fig. 5(a). For the three tapes, the local resonance frequencies correspond to the theoretical $S_{1}^{\prime} S_{2}^{\prime}$ - and $A_{2}^{\prime} A_{3}^{\prime}-\mathrm{ZGV}$ resonances frequencies calculated for a material of Poisson's ratio $\nu=0.332$ [Fig. 5(b)], which is in good agreement with values generally found in the literature for aluminum alloys.

In order to confirm the origin of the resonances, the source was translated along the strip over $350 \mathrm{~mm}$ to observe the different modes. A Bscan $u_{y}(x, t)$ was recorded for $3 \mathrm{~ms}$ [Fig. $6(\mathrm{a})$. The signal is dominated by the pseudo-Rayleigh waves (edge wave), and the first reflections at the tape end appear after $400 \mu \mathrm{s}$. An average of two-dimensional Fourier transforms calculated on $450-\mu$ s sliding time windows provides the dispersion curves. Several modes are measured up to a frequency $\times$ width product of $f b=4 V_{T}$. The $S_{1}^{\prime} S_{2}^{\prime}$ - and $A_{2}^{\prime} A_{3}^{\prime}$ $\mathrm{ZGV}$ points just below the cutoff frequencies $f b=V_{T}$ and $f b=1.5 V_{T}$, as well as the associated backward modes, are

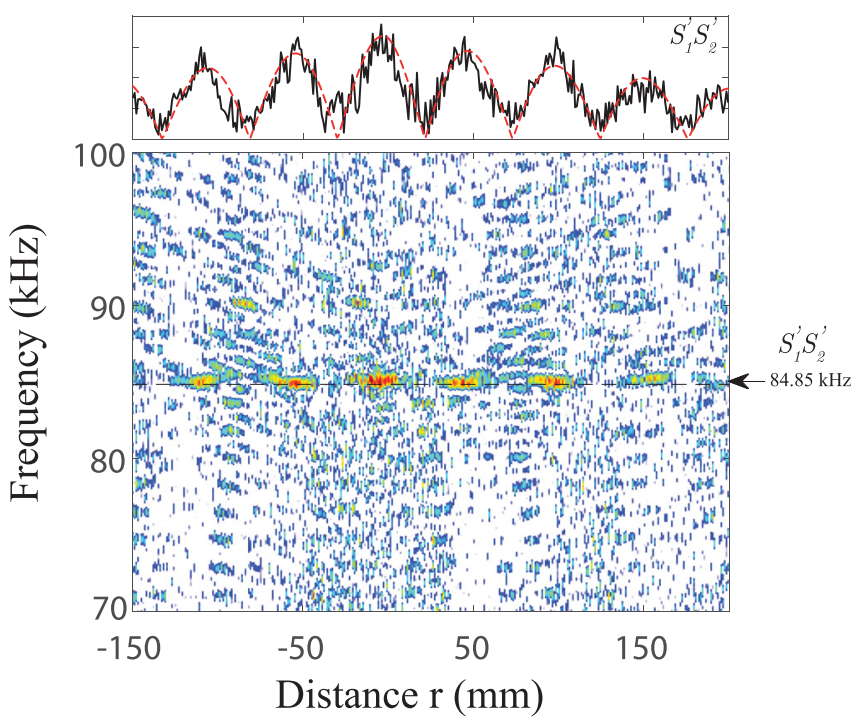

FIG. 7. (Color online) Spatial distribution of the $u_{y}$ measured displacement around the $S_{1}^{\prime} S_{2}^{\prime}-\mathrm{ZGV}$ frequency. Top curves represent amplitude profile at the frequency $84.85 \mathrm{kHz}$ (black) and the theoretical damped cosine profile (red dashed line). 
clearly identified and in good agreement with the theoretical curves. The temporal Fourier transform of the displacement profile $u_{y}(x, f)$ is displayed around the $S_{1}^{\prime} S_{2}^{\prime}-\mathrm{ZGV}$ frequency (Fig. 7). The interference pattern of the $\mathrm{ZGV}$ modes is clearly observed at $f=84.85 \mathrm{kHz}$ and shown in Fig. 7(a). The red dashed line is the damped cosine function $\left|\cos \left(2 \pi x / \lambda_{S_{1}^{\prime} S_{2}^{\prime}}\right)\right|$ $\times \exp (-\alpha|x|)$, where the $\mathrm{ZGV}$ mode wavelength is $\lambda_{S_{1}^{\prime} S_{2}^{\prime}}$ $=103 \mathrm{~mm}$, and $\alpha$ is an adjusted damping parameter. This profile is in good agreement with the numerical displacements field that will be shown in the Appendix.

\section{HARD AND SOFT RIBBON: COMPARISON THROUGH NUMERICAL ANALYSIS}

In order to complete these measurements, the finitedifference time domain code Simsonic ${ }^{48}$ was used to simulate the propagation of low frequency in-plane modes in a thin tape. The first calculation was achieved for the duraluminum tape, which is similar to the one in the experiments ( $L=10 \mathrm{~m}, b=30 \mathrm{~mm}$, and $d=2 \mathrm{~mm}$ ). Then, we calculated the waves propagating in a soft ribbon of Poisson's ratio $\nu \approx 0.5$ with $V_{T}=5 \mathrm{~m} / \mathrm{s}, V_{L}=1500 \mathrm{~m} / \mathrm{s}$, and geometrical parameters $L=2 \mathrm{~m}, b=20 \mathrm{~mm}$, and $d=2 \mathrm{~mm}$. The strips lengths were chosen large enough to avoid mode reflections at the end.

In order to excite in-plane modes, a normal velocity source with a constant profile along the thickness and a Gaussian profile along the $x$ axis was applied on one tape edge. Then the velocity field $V_{y}(t, x, b / 2,0)$ was registered along the opposite edge for $-1<x<1 \mathrm{~m}$, for $2 \mathrm{~ms}$ for the metal tape and $200 \mathrm{~ms}$ for the soft ribbon. It is represented as a function of dimensionless time $t V_{T} / b$ and distance $x / b$ [Figs. 8(a) and 8(d)]. In both Bscans, it can be observed that after two or three reflections of the $S_{0}$ mode, the stationary modes appear.

The 2D-Fourier transform of the field $V_{y}(t, x, b / 2,0)$, $x>0$ is calculated and displayed with the theoretical dispersion curves calculated with the MK model [Figs. 8(b) and 8(e)]. The modes having a nonzero velocity component $V_{y}$ are observed and in good agreement with the theoretical modes. For the solid strip, the two backward modes $S_{2 b}^{\prime}$ and $A_{2 b}^{\prime}$ are clearly detected. In the soft strip, the well generated $S_{2 b}^{\prime}$ backward mode appears as the continuation of the $S_{2}^{\prime}$ mode, confirming the existence of a Dirac cone.

The frequency spectra of the velocity recorded at point $(x, y, z)=(0,-b / 2,0)$ opposite to the source center are
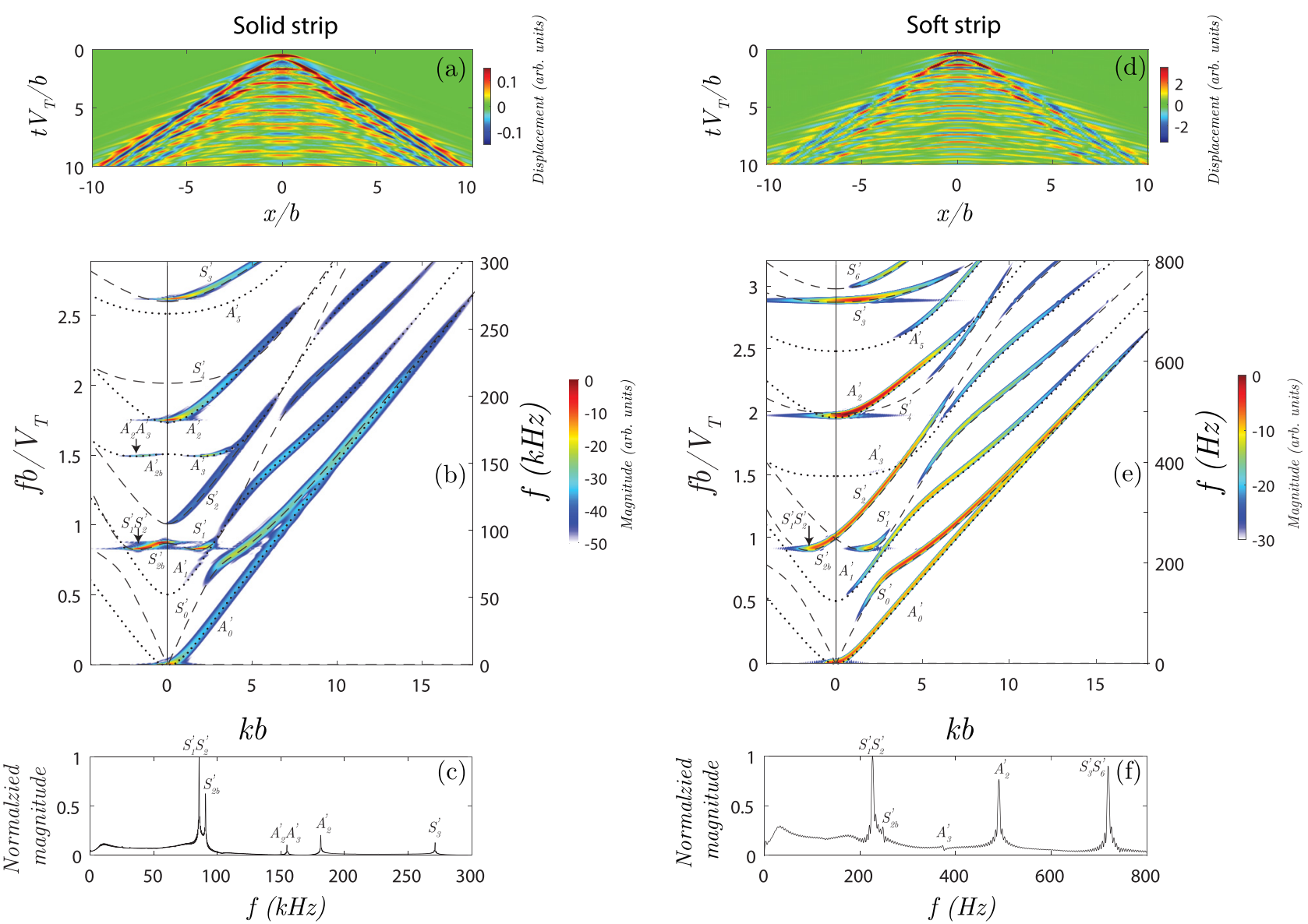

FIG. 8. (Color online) FDTD simulation of in-plane mode propagation in a duraluminum tape of parameters $L=10 \mathrm{~m}, b=30 \mathrm{~mm}, d=2 \mathrm{~mm}, V_{L}=6400 \mathrm{~m} / \mathrm{s}$, $V_{T}=3140 \mathrm{~m} / \mathrm{s}$ (left), and a soft ribbon of parameters $L=2 \mathrm{~m}, b=20 \mathrm{~mm}, d=2 \mathrm{~mm}, V_{L}=1500 \mathrm{~m} / \mathrm{s}, V_{T}=5 \mathrm{~m} / \mathrm{s}$ (right): normal edge velocity as a function of dimensionless time and distance [(a) and (d)], 2D-Fourier transform of the normal edge velocity with superimposed theoretical dispersion curves obtained with the MK model [(b) and (e)], local resonances recorded at point $(x, y, z)=(0,-b / 2,0)$ opposite to the source center [(c) and (f)]. 
plotted in Figs. 8(c) and 8(f). For the solid strip, the three resonances are associated to the $S_{0}$ mode cutoff of $S_{2 b}^{\prime}, A_{2}^{\prime}$ and $S_{3}^{\prime}$, the other two are the $S_{1}^{\prime} S_{2}^{\prime}$ - and $A_{2}^{\prime} A_{3}^{\prime}-\mathrm{ZGV}$ resonances that were measured in the experiment. The cutoff frequency resonances are well excited in this numerical simulation because the source is normal to the edge, which is in favor the $S_{0}$ plate mode generation, while a laser source in the thermoelastic regime mostly generate a shear wave. ${ }^{49}$ For the soft strip, the $S_{1}^{\prime} S_{2}^{\prime}$ - and $S_{3}^{\prime} S_{6}^{\prime}$-ZGV resonances and the $A_{2}^{\prime}$ cutoff resonance dominate. On the contrary, there is no significant resonance at the $S_{2}^{\prime}$ (or $S_{2 b}^{\prime}$ ) cutoff frequency. In fact, as indicated by Mindlin, ${ }^{5,6}$ at the Dirac cone, the group velocity remains finite and equal to $V_{g}=2 V_{T} / \pi$, so that all modes are propagative and no resonance occurs.

\section{CONCLUSION}

The propagation of in-plane modes in thin rectangular beams corresponding to low frequency longitudinal $L$-modes and bending $B_{x}$-modes was studied. Dispersion curves were simulated and measured in metallic tape. A good agreement was observed with theoretical dispersion curves calculated with the MK model. The backward modes and the associated $\mathrm{ZGV}$ mode resonances were clearly observed. We showed that these resonances do not depend on the strip thickness, and the measurement of the two lowest $\mathrm{ZGV}$ resonances can be used to determine the strip material Poisson's ratio $\nu$. Nondissipative soft ribbons were then considered, showing that backward modes exist at frequencies very low compared to the longitudinal cutoff frequency. These backward modes, weakly coupled to the embedding fluid, could occur in the tectorial membrane, for example, and the associated in-plane displacements may play a role in the complex audition mechanism. However, theoretical studies should be conducted to account for viscoelastic properties, and new experiments should be developed to measure guided waves in soft strips. As already observed for Lamb waves, we expect that backward and forward branches will split at the $\mathrm{ZGV}$ point, and the Dirac cone, associated to a finite energy velocity, will remain.

\section{ACKNOWLEDGMENTS}

The authors wish to thank B. Gérardin for drawing our attention to the Santamore and Cross paper (Ref. 1) and A. A. Krushynska for fruitful discussions on dispersion curves in elastic bars of rectangular cross section. The authors are grateful for funding provided by LABEX WIFI (Laboratory of Excellence within the French Program Investments for the Future, Grant No. ANR-10-LABX-24) and Grant No. ANR-10-IDEX-0001-02 PSL*.

\section{APPENDIX: DISPLACEMENT FIELD AT ZGV RESONANCE FREQUENCIES}

For the duraluminum, the velocity field $\left(v_{x}, v_{y}\right)$ was also recorded in the plane $z=0$, and the in-plane displacement field was deduced by numerical integration. After a temporal Fourier transform of a $1 \mathrm{~ms}$ signal, the in-plane displacement distribution was observed at the first two ZGV frequencies [Figs. 9(a)-9(d)]. The displacement components $u_{x}$ and $u_{y}$ are of the same order of magnitude and similar to those of the ZGV Lamb modes. ${ }^{15}$

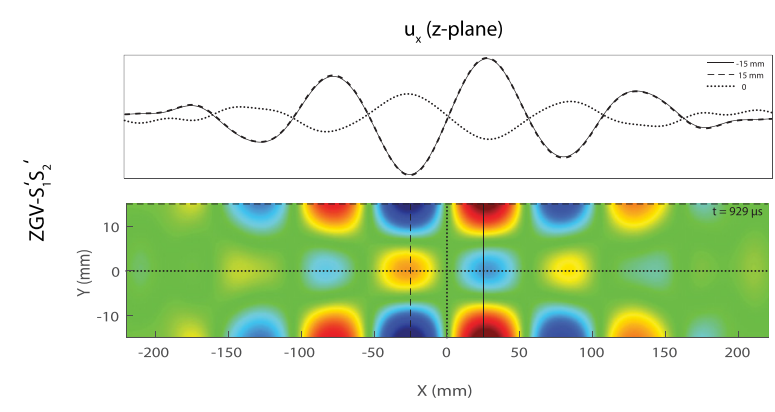

(a)

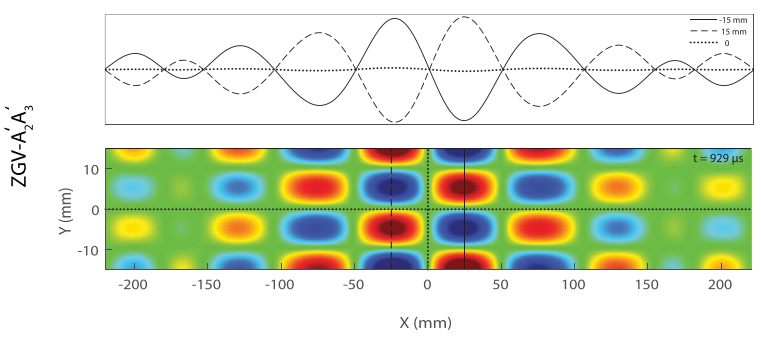

(b)

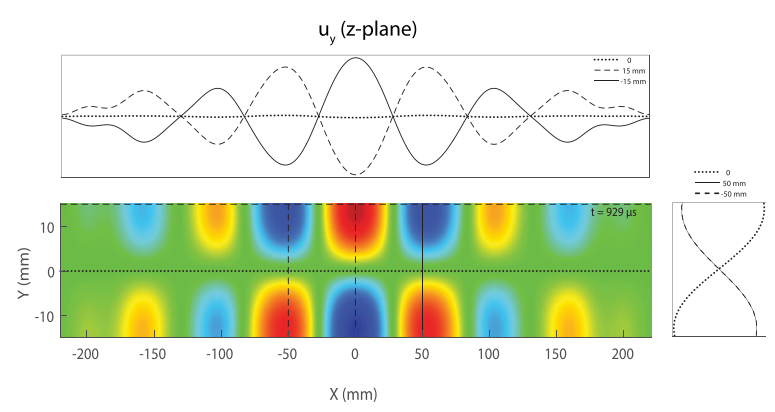

(c)

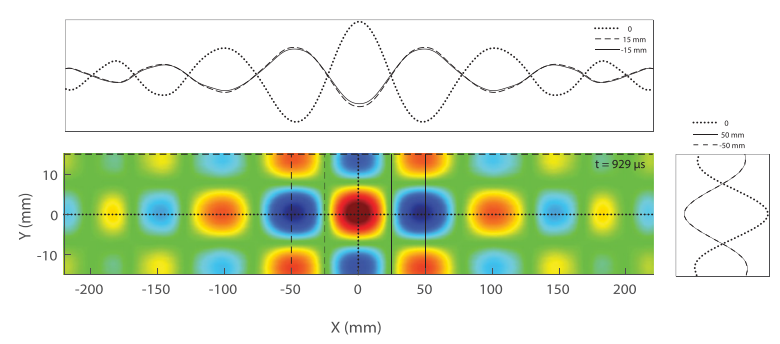

(d)

FIG. 9. (Color online) In-plane displacement components at the $S_{1}^{\prime} S_{2}^{\prime}$-ZGV frequency $u_{x}$ (a) and $u_{y}$ (c) and at the $A_{2}^{\prime} A_{3}^{\prime}$-ZGV frequency $u_{x}$ (b) and $u_{y}$ (d) simulated with the elastodynamic finite-difference time domain (FDTD) code Simsonic for a duraluminum tape of aspect ratio $b / d=15$. 
${ }^{1}$ D. H. Santamore and M. C. Cross, "Surface scattering analysis of phonon transport in the quantum limit using an elastic model," Phys. Rev. B. 66, 144302 (2002).

${ }^{2}$ C. Jean, L. Belliard, L. Becerra, and B. Perrin, "Backward propagating acoustic waves in single gold nanobeams," Appl. Phys. Lett. 107, 193103 (2015).

${ }^{3}$ J. Brum, M. Bernal, J. Gennisson, and M. Tanter, "In vivo evaluation of the elastic anisotropy of the human Achilles tendon using shear wave dispersion analysis," Phys. Med. Biol. 59, 505-523 (2014).

${ }^{4}$ J. B. Sellon, S. Farrahi, R. Ghaffari, and D. M. Freeman, "Longitudinal spread of mechanical excitation through tectorial membrane traveling waves," Proc. Natl. Acad. Sci. U.S.A. 112, 12968-12973 (2015).

${ }^{5}$ R. D. Mindlin, "Mathematical theory of vibrations of elastic plates," 11th Annual Symposium on Frequency Control (IEEE, 1957), pp. 1-40.

${ }^{6} \mathrm{R}$. D. Mindlin, An introduction to the mathematical theory of vibrations of elastic plates, edited by J. Yang (World Scientific, Singapore, 2006).

${ }^{7} \mathrm{~A}$. Meitzler, "Backward-wave transmission of stress pulses in elastic cylinders and plates," J. Acoust. Soc. Am. 38, 835-840 (1965).

${ }^{8}$ J. Wolf, T. D. K. Ngoc, R. Kille, and W. G. Mayer, "Investigation of Lamb waves having a negative group velocity," J. Acoust. Soc. Am. 83, 122 (1988).

${ }^{9}$ P. Burlii and I. Y. Kucherov, "Backward elastic waves in plates," JETP Lett. 26, 644-647 (1977).

${ }^{10} \mathrm{C}$. Prada, O. Balogun, and T. Murray, "Laser based ultrasonic generation and detection of zero-group velocity Lamb waves in thin plates," Appl. Phys. Lett. 87, 194109 (2005).

${ }^{11}$ B. Zaitsev, I. Kuznetsova, I. Nedospasov, A. Smirnov, and A. Semyonov, "New approach to detection of guided waves with negative group velocity: Modeling and experiment," J. Sound Vib. 442, 155-166 (2019).

${ }^{12}$ I. Tolstoy and E. Usdin, "Wave propagation in elastic plates: Low and high mode dispersion," J. Acoust. Soc. Am. 29, 37-42 (1957).

${ }^{13}$ D. Holland and D. E. Chimenti, "Air-coupled acoustic imaging with zerogroup-velocity Lamb modes," Appl. Phys. Lett. 83, 2704-2706 (2003).

${ }^{14}$ V. Yantchev, L. Arapan, I. Katardjiev, and V. Plessky, "Thin-film zerogroup-velocity Lamb wave resonator," Appl. Phys. Lett. 99, 033505 (2011).

${ }^{15} \mathrm{O}$. Balogun, T. Murray, and C. Prada, "Simulation and measurement of the optical excitation of the $\mathrm{S} 1$ zero group velocity Lamb wave resonance in plate," J. App. Phys. 102, 064914 (2007).

${ }^{16}$ D. Clorennec, C. Prada, D. Royer, and T. W. Murray, "Laser impulse generation and interferometer detection of zero group velocity Lamb mode resonance," Appl. Phys. Lett. 89, 024101 (2006).

${ }^{17}$ C. Grünsteidl, T. W. Murray, T. Berer, and I. A. Veres, "Inverse characterization of plates using zero group velocity Lamb modes," Ultrasonics 65, 1-4 (2016).

${ }^{18}$ Q. Xie, S. Mezil, P. H. Otsuka, M. Tomoda, J. Laurent, O. Matsuda, Z. Shen, and O. B. Wright, "Imaging gigahertz zero-group-velocity Lamb waves," Nat. Comm. 10, 2228 (2019).

${ }^{19}$ D. Clorennec, C. Prada, and D. Royer, "Local and noncontact measurements of bulk acoustic wave velocities in thin isotropic plates and shells using zero group velocity Lamb modes," J. Appl. Phys. 101, 034908 (2007).

${ }^{20}$ M. Cès, D. Clorennec, D. Royer, and C. Prada, "Thin layer thickness measurements by zero group velocity Lamb mode resonances," Rev. Sci. Instr. 82, 114902 (2011).

${ }^{21}$ J. Laurent, D. Royer, T. Hussain, F. Ahmad, and C. Prada, "Laser induced zero-group velocity resonances in transversely isotropic cylinder," J. Acoust. Soc. Am. 137, 3325-3334 (2015).

${ }^{22}$ D. Mounier, C. Poilâne, H. Khelfa, and P. Picart, "Sub-gigahertz laser resonant ultrasound spectroscopy for the evaluation of elastic properties of micrometric fibers," Ultrasonics 54, 259-267 (2014).

${ }^{23}$ R. Mindlin and E. Fox, "Vibrations and waves in elastic bars of rectangular cross section," J. Appl. Mech. 27, 152-158 (1960).

${ }^{24} \mathrm{R}$. Morse, "The velocity of compressional waves in rods of rectangular cross section,” J. Acoust. Soc. Am. 22, 219-223 (1950).
${ }^{25}$ M. A. Medick, "Extensional waves in elastic bars of rectangular cross section," J. Acoust. Soc. Am. 43, 152-161 (1968).

${ }^{26}$ O. M. Mukdadi, Y. Desai, S. Datta, A. Shah, and A. Niklasson, "Elastic guided waves in a layered plate with rectangular cross section," J. Acoust. Soc. Am. 112, 1766-1779 (2002).

${ }^{27}$ O. M. Mukdadi and S. K. Datta, "Transient ultrasonic guided waves in layered plates with rectangular cross section," J. Appl. Phys. 93, 9360-9370 (2003).

${ }^{28} \mathrm{~N}$. Stephen and P. Wang, "Saint-Venant decay rates for the rectangular cross section rod," J. Appl. Mech. 71, 429-433 (2004).

${ }^{29}$ D. H. Cortes, S. K. Datta, and O. M. Mukdadi, "Elastic guided wave propagation in a periodic array of multi-layered piezoelectric plates with finite cross-sections," Ultrasonics 50, 347-356 (2010).

${ }^{30} \mathrm{~F}$. Cegla, "Energy concentration at the center of large aspect ratio rectangular waveguides at high frequencies," J. Acoust. Soc. Am. 123, 4218-4226 (2008).

${ }^{31}$ R. Kažys, E. Žukauskas, L. Mažeika, and R. Raišutis, "Propagation of ultrasonic shear horizontal waves in rectangular waveguides," Int. J. Struct. Stab. Dyn. 16, 1550041 (2016).

${ }^{32} \mathrm{~V}$. Meleshko, A. Bondarenko, A. Trofimchuk, and R. Abasov, "Elastic waveguides: History and the state of the art. II," J. Math. Sci. 167, 197-216 (2010).

${ }^{33}$ A. Krushynska and V. Meleshko, "Normal waves in elastic bars of rectangular cross section," J. Acoust. Soc. Am. 129, 1324-1335 (2011).

${ }^{34} \mathrm{R}$. W. Morse, "Dispersion of compressional waves in isotropic rods of rectangular cross section," J. Acoust. Soc. Am. 20, 833-838 (1948).

${ }^{35} \mathrm{P}$. Hertelendy, "An approximate theory governing symmetric motions of elastic rods of rectangular or square cross section," J. Appl. Mech. 35, 333-341 (1968).

${ }^{36}$ I. A. Veres and M. B. Sayir, "Wave propagation in a wooden bar," Ultrasonics 42, 495-499 (2004).

${ }^{37}$ V. Serey, N. Quaegebeur, P. Micheau, P. Masson, M. Castaings, and M. Renier, "Selective generation of ultrasonic guided waves in a bidimensional waveguide," Struct. Health Monit. 18(4), 1324-1336 (2019).

${ }^{38}$ D. Royer and E. Dieulesaint, Elastic Waves in Solids I: Free and Guided Propagation (Springer, 1999).

${ }^{39}$ M. Medick and Y.-H. Pao, "Extensional vibrations of thin rectangular plates," J. Acoust. Soc. Am. 37, 59-65 (1965).

${ }^{40} \mathrm{~W}$. Fraser, "Stress wave propagation in rectangular bars," Int. J. Sol. Struct. 5, 379-397 (1969).

${ }^{41} \mathrm{G}$. Kynch, "The fundamental modes of vibration of uniform beams for medium wavelengths,” Br. J. Appl. Phys. 8, 64 (1957).

${ }^{42}$ A. Sarvazyan, "Low-frequency acoustic characteristics of biological tissues," Poly. Mech. 11, 594 (1975).

${ }^{43}$ C. Prada, D. Clorennec, and D. Royer, "Power law decay of zero group velocity Lamb modes," Wave Motion 45, 723-728 (2008).

${ }^{44}$ A. Maznev, "Dirac cone dispersion of acoustic waves in plates without phononic crystals," J. Acoust. Soc. Am. 135, 577-580 (2014).

${ }^{45}$ D. M. Stobbe and T. W. Murray, "Conical dispersion of Lamb waves in elastic plates,” Phys. Rev. B. 96, 144101 (2017).

${ }^{46}$ Optimal source length at constant maximum surface energy density: The absorbed energy distribution is written as $\boldsymbol{E}(x)=I \exp \left(-x^{2} / l^{2}\right)$, where $I$ is supposed to be lower than the ablation threshold. The resulting Fourier transform is given by $\boldsymbol{E}(k)=(I \sqrt{\pi} l) \times \exp \left(-l^{2} k^{2} / 4\right)$. The amplitude of a mode of wave number $\mathrm{k}$ reaches a maximum for the optimal source length $l_{\text {opt }}=\lambda /(\pi \sqrt{2})$.

${ }^{47}$ A. Jia, "Laser-generated pseudo-Rayleigh acoustic wave propagating along the edge of a thin plate," J. Appl. Phys. 80, 2595 (1996).

${ }^{48}$ E. Bossy, M. Talmant, and P. Laugier, "Three-dimensional simulations of ultrasonic axial transmission velocity measurement on cortical bone models," J. Acoust. Soc. Am. 115, 2314 (2004).

${ }^{49}$ D. Hutchins, R. Dewhurst, and S. Palmer, "Directivity patterns of lasergenerated ultrasound in aluminum," J. Acoust. Soc. Am. 70, 1362-1369 (1981). 